Article

\title{
A Study on Controllability of a Class of Impulsive Fractional Nonlinear Evolution Equations with Delay in Banach Spaces
}

\author{
Daliang Zhao
}

School of Mathematics and Statistics, Shandong Normal University, Jinan 250014, China; dlzhao928@sdnu.edu.cn

check for updates

Citation: Zhao, D. A Study on Controllability of a Class of Impulsive Fractional Nonlinear Evolution Equations with Delay in Banach Spaces. Fractal Fract. 2021, 5, 279. https://doi.org/10.3390/ fractalfract5040279

Academic Editor: Omar Bazighifan

Received: 3 November 2021

Accepted: 13 December 2021

Published: 17 December 2021

Publisher's Note: MDPI stays neutral with regard to jurisdictional claims in published maps and institutional affiliations.

Copyright: (C) 2021 by the author. Licensee MDPI, Basel, Switzerland. This article is an open access article distributed under the terms and conditions of the Creative Commons Attribution (CC BY) license (https:// creativecommons.org/licenses/by/ $4.0 /)$.

\begin{abstract}
Under a new generalized definition of exact controllability we introduced and with a appropriately constructed time delay term in a special complete space to overcome the delayinduced-difficulty, we establish the sufficient conditions of the exact controllability for a class of impulsive fractional nonlinear evolution equations with delay by using the resolvent operator theory and the theory of nonlinear functional analysis. Nonlinearity in the system is only supposed to be continuous rather than Lipschitz continuous by contrast. The results obtained in the present work are generalizations and continuations of the recent results on this issue. Further, an example is presented to show the effectiveness of the new results.
\end{abstract}

Keywords: controllability; impulsive fractional evolution equations; delay; measure of noncompactness; mild solution; fixed point theorem

\section{Introduction}

This paper's primary objective is to investigate the exact controllability of the following impulsive fractional nonlinear evolution equations with delay in Banach spaces:

$$
\left\{\begin{array}{l}
D^{\gamma} x(t)=A x(t)+f\left(t, x(t), x_{t}\right)+B u(t), \text { a.e. } t \in I:=[0, a] \\
\Delta x\left(t_{i}\right)=x\left(t_{i}^{+}\right)-x\left(t_{i}^{-}\right)=I_{i}\left(x\left(t_{i}\right)\right), \quad i=1,2, \cdots, m \\
x(t)=\phi(t), \quad t \in[-b, 0]
\end{array}\right.
$$

where $D^{\gamma}$ represents the Caputo derivative of order $\gamma \in(0,1)$. The state $x(\cdot)$ takes values in $X$, control function $u$ is given in $L^{2}(I, U)$, and $B: U \rightarrow X$ is a bounded linear operator where $X$ and $U$ are Banach spaces. $0=t_{0}<t_{1}<t_{2}<\cdots<t_{m}<t_{m+1}=a<+\infty$. $A: \mathcal{D} \subset X \rightarrow X$ is a closed linear unbounded operator on $X$ with dense domain $\mathcal{D}$. $x_{t}$ represents the history of the state function that will be specified in (2). $\phi(t) \in C([-b, 0], X)$. The given functions $f$ and $I_{i}(i=1,2, \cdots, m)$ are supposed to satisfy some appropriate assumptions that will be specified later.

In the last few decades, the topic of fractional calculus has received considerable and extensive attention. The modelling of many mathematical and biological problems by fractional differential equations has more superiority and accuracy than classical integral-order ones. In view of its extensive applications in the area of physics, chemistry, mathematics, medicine and economics, a growing number of researchers have devoted generous energy to the study of various types of fractional differential equations. For further details of the recent works, we refer readers to [1-5].

It is well known that impulse and delay embody lots of rich and varied dynamic behaviors. The investigation of various dynamical systems with impulsive interference and time delay effects has obtained more and more attention due to their important and potential applications in signal and image processing, weather predicting, artificial intelligence and some other optimization problems. For more details, one can see [6-8].

It is noted that the research on the controllability of fractional differential equations is becoming more and more active, since controllability is a quite important concept in mathematics and control theory. As one of the most mainstream research direction, exact 
controllability of many kinds of integral-order and fractional-order control systems have been well investigated by taking advantage of diverse tools and methods in some recent literatures. For example, S. Ji et al. [9] studied the exact controllability of a class of integralorder impulsive differential equations by using the measure of noncompactness and fixed point theorem under a compact condition imposed on the nonlocal item. J. Wang and Y. Zhou [10] investigated a class of fractional differential systems without assuming the compactness of the semigroup. They discussed the exact controllability of the considered control systems under the assumption that the nonlinearity satisfied Lipschitz continuity. In [11], J. Du et al. obtained a result of exact controllability for some fractional neutral integro-differential evolution systems with delay and nonlocal conditions. The Lipschitz condition and some other growth conditions on nonlocal item and nonlinearity are still necessary. Z. Tai [12] proved the exact controllability results for fractional impulsive neutral integro-differential systems in Banach spaces. The results are obtained by utilizing Banach contraction mapping theorem due to the Lipschitz conditions of the systems. In addition, some excellent results of exact controllability for various fractional differential equations have also been established recently [7,10-23], but the limitation is also that the functions in the systems are either Lipschitz continuous, compact or satisfy some special growth suppositions. Although the exact controllability studied in [13] does not require the nonlinear term to satify Lipschitz condition, the considered evolution system in [13] have no effects of time delay and impulse. At present, it seems that the exact controllability results of fractional evolution equations with both impulses and delays are rare $[12,21,22]$. We point out that nonlinearities and impulsive items in these papers satisfy special growth assumptions [21], Lipschitz condition [12,22], and semigroups together with the resolvent operators of some systems possess compactness, which still show the limitation to a certain extent in practical problems. Therefore, it seems interesting whether the exact controllability of the impulsive fractional evolution equations with delay can be established via noncompact resolvent operators together with the nonlinearity satisfying continuity rather than Lipschitz continuity.

Inspired by the abovementioned papers and the ideas adopted in [13], in this work, we present a new depiction of the exact controllability of the system (1) by using the theory of resolvent operator and the theory of nonlinear functional analysis. The main contributions of this article are as follows. (i) The Lipschitz and other restrictive conditions on nonlinear and impulsive items have been removed. (ii) The application of $C_{0}$-semigroup based on probability density function [24] is replaced by resolvent operators without compact conditions, which is different from most of the existing literatures such as $[7,10-12,17,21,22,25,26]$. (iii) With the properly defined delay item in a corresponding complete space we introduced, we have solved the delay-induced-difficulty during the investigation of exact controllability by measures of noncompactness.

The organization of this work is as follows. Some necessary notations, definitions and lemmas are introduced in next section. In the third section, sufficient conditions ensuring exact controllability of the addressed systems are provided. An example is worked out in the last section to illustrate our theory of the main results.

\section{Preliminaries}

We denote by $X$ a Banach space with the norm $\|\cdot\|$. By $C(I, X)$ and $C([-b, a], X)$ we denote the spaces of continuous functions from $I$ into $X,[-b, a]$ into $X$ with suprema norms $\|\cdot\|_{C(I, X)}$ and $\|\cdot\|_{C([-b, a], X)}$, respectively. For the case of $a=0$, norm $\|\cdot\|_{C([-b, a], X)}$ is abbreviated as $\|\cdot\|_{b}$. Also consider the usual Banach space $P C(I, X)=\{x: I \rightarrow X \mid$ $x \in C\left(\left(t_{k}, t_{k+1}\right], X\right), x\left(t_{k}^{-}\right)$and $x\left(t_{k}^{+}\right)$exist with $\left.x\left(t_{k}^{-}\right)=x\left(t_{k}\right), k=1,2, \cdots, m\right\}$, with the norm $\|x\|_{P C}=\sup _{t \in I}\{\|x(t)\|\}$. D stands for the domain of the operator $A$ in (1) with the graph norm $\|x\|_{\mathcal{D}}^{t \in I}=\|x\|+\|A x\|$. Denote by $U$ a Banach space with the norm $\|\cdot\|_{U}$. By $C^{\gamma}(I, X), \gamma \in(0,1)$, we denote the space of all the $\gamma$-Hölder continuous functions 
from $I$ into $X$ with the norm $\|x\|_{C^{\gamma(I, X)}}=\|x\|_{C(I, X)}+[|x|]_{C^{\gamma(I, X)}}$, where $[|x|]_{C^{\gamma(I, X)}}=$ $\sup _{t, s \in I, t \neq s} \frac{\|x(t)-x(s)\|}{(t-s)^{\gamma}}$. For any measurable function $x: I \rightarrow R$, define the norm

$$
\|x\|_{L^{p}(I)}= \begin{cases}\left(\int_{I}|x(t)|^{p} d t\right)^{\frac{1}{p}}, & 1 \leq p<\infty, \\ \inf _{\mu(\bar{I})=0}\left\{\sup _{t \in I-\bar{I}}|x(t)|\right\}, & p=\infty,\end{cases}
$$

where $\mu(\bar{I})$ is the Lebesgue measure on $\bar{I}$. Let $\mathcal{L}(X, Y)$ be the space of all bounded linear operators from $X$ into Banach space $Y$ equipped with operator norm $\|\cdot\|_{\mathcal{L}(X, Y)}$.

Introduce a complete and integrable space $L([-b, 0], X)$ which contains all the integrable functions from $[-b, 0]$ into $X$. For $x \in P C(I, X)$ and $t \in I$, define a piecewise function as follows:

$$
x_{t}(\theta)= \begin{cases}x(t+\theta), & t+\theta \geq 0, \\ \phi(t+\theta), & t+\theta \leq 0,\end{cases}
$$

for every $\theta \in[-b, 0]$, where $\phi$ is the same as in (1). It is not hard to verify that $x_{t} \in L([-b, 0], X)$.

Remark 1. Based on (2) and Lemma 4 together with Lemma 5 which we will present in the following discussions, it is much more convenient to study the exact controllability of system (1) by using the theory of noncompact measures.

Next we list the well known definitions as follows.

Definition 1. ([27]) The fractional integral with order $\gamma>0$ for a function $u:(0,+\infty) \rightarrow R$ can be defined as

$$
I_{0^{+}}^{\gamma} u(t)=\frac{1}{\Gamma(\gamma)} \int_{0}^{t}(t-s)^{\gamma-1} u(s) d s,
$$

provided that the right side integral is pointwise defined on $[0,+\infty)$.

Definition 2. ([27]) The Caputo fractional derivative with order $\gamma>0$ for a function $u:(0, \infty) \rightarrow$ $R$ is written as

$$
D_{0^{+}}^{\gamma} u(t)=\frac{1}{\Gamma(n-\gamma)} \int_{0}^{t} \frac{u^{(n)}(s)}{(t-s)^{\gamma-n+1}} d s,
$$

where $n=[\gamma]+1$, provided the right side integral is pointwise defined on $[0, \infty)$.

Definition 3. ([28]) A family of bounded linear operators $\{\wp(t)\}_{t \geq 0} \subset \mathcal{L}(X)$ on $X$ is called a resolvent operator of integral equation

$$
x(t)=\int_{0}^{t} \frac{(t-s)^{\gamma-1}}{\Gamma(\gamma)} A x(s) d s, t \geq 0,
$$

if the following assumptions are satisfied:

(i) $\wp(t)$ is strongly continuous on $R^{+}$and $\wp(0)=I$;

(ii) $\wp(t) \mathcal{D} \subset \mathcal{D}, A \wp(t) x=\wp(t) A x$ for every $t \geq 0$ and $x \in \mathcal{D}$;

(iii) the resolvent equation holds

$$
\wp(t) x=x+\int_{0}^{t} \frac{(t-s)^{\gamma-1}}{\Gamma(\gamma)} A \wp(s) x d s .
$$


Definition 4. ([28]) A resolvent operator $\wp(t)$ of (3) is called differentiable, if there is a function $\varphi \in L_{\text {loc }}^{1}\left(R^{+}\right)$such that the following inequality holds:

$$
\|\dot{\wp}(t) x\| \leq \varphi(t)\|x\|_{\mathcal{D}} \text { a.e. on } R^{+}, \forall x \in \mathcal{D},
$$

where $\wp(\cdot) x \in W_{\text {loc }}^{1,1}\left(R^{+}, X\right)$ for each $x \in \mathcal{D}$.

Consider the equation

$$
x(t)=g(t)+\int_{0}^{t} \frac{(t-s)^{\gamma-1}}{\Gamma(\gamma)} A x(s) d s, \quad t \in I,
$$

where $g \in L^{1}(I, X)$. According to [28], the mild solution of (4) can be defined as follows.

Definition 5. We call $x \in C(I, X)$ a mild solution for (4) if $\int_{0}^{t} \frac{(t-s)^{\gamma-1}}{\Gamma(\gamma)} x(s) d s \in \mathcal{D}$, and satisfies

$$
x(t)=g(t)+A \int_{0}^{t} \frac{(t-s)^{\gamma-1}}{\Gamma(\gamma)} x(s) d s
$$

for each $t \in I$.

Now, let us give a useful lemma about differentiable resolvent operator from which one can get the equivalent definition of mild solution for Equation (4).

Lemma 1. ([28]) Assume that $\wp(t)$ is a differentiable resolvent operator for (4) and $g \in C(I, \mathcal{D})$. Then

$$
x(t)=\int_{0}^{t} \dot{\wp}(t-s) g(s) d s+g(t), \quad t \in I,
$$

is a mild solution of (4).

We now recall some useful properties of Kuratowski measures of noncompactness. For more details, please refer [29].

Lemma 2. Let $X$ be a Banach space and $\beta(\cdot)$ be the Kuratowski measures of noncompactness which is given by $\beta(\Omega)=\inf \left\{\delta>0: \Omega=\bigcup_{i=1}^{k} \Omega_{i}\right.$ with $\left.\operatorname{diam}\left(\Omega_{i}\right) \leq \delta, i=1,2, \cdots, k\right\}$ for a bounded subset $\Omega$ in $X$.

(I) Let $D_{1}, D_{2}$ be bounded sets of $X$ and $\lambda \in R$. Then

(i) $\beta\left(D_{1}\right)=0 \Leftrightarrow D_{1}$ is relatively compact;

(ii) $\beta\left(\lambda D_{1}\right)=|\lambda| \beta\left(D_{1}\right)$;

(iii) $\beta\left(D_{1}+D_{2}\right) \leq \beta\left(D_{1}\right)+\beta\left(D_{2}\right)$;

(II) Assume that $D=\left\{u_{n}\right\}$ is a countable set of strongly measurable functions from I into Banach space $X$, and there has a function $\psi \in L^{1}(I)$ such that $\left\|u_{n}(t)\right\| \leq \psi(t)$ a.e.t $\in I, n=1,2, \cdots$, then $\beta(D(t))$ is integrable on $I$, and satisfies

$$
\beta\left(\left\{\int_{I} u_{n}(t) d t: n \in N\right\}\right) \leq 2 \int_{I} \beta(D(t)) d t .
$$

For convenience, the Kuratowski measures of noncompactness of a bounded subset in spaces $X, P C(I, X)$ and $L([-b, 0], X)$ are all denoted by $\beta(\cdot)$, on the premise of no confusion.

Lemma 3. (Mönch) Suppose $X$ to be a Banach space and $D \subset X$ is a closed and convex set, $x_{0} \in D$. If $A: D \rightarrow D$ is continuous and satisfies: $C \subset D$ countable, $C \subset \overline{c o}\left(\left\{x_{0}\right\} \cup A(C)\right) \Rightarrow C$ is relatively compact. Then $A$ has a fixed point in $D$.

At last of this section, we present two important lemmas as follows. 
Lemma 4. Suppose that $x_{n}$ converges to $x_{0}$ in $P C(I, X)$ as $n \rightarrow+\infty$. Then $\left(x_{n}\right)_{t}$ converges to $\left(x_{0}\right)_{\text {t }}$ in $L([-b, 0], X)$ for each $t \in I$ as $n \rightarrow+\infty$.

Proof. By (2), we can obtain

(i) if $t \leq b$, then

$$
\begin{aligned}
\left\|\left(x_{n}\right)_{t}-\left(x_{0}\right)_{t}\right\|_{L[-b, 0]} & =\int_{-b}^{0}\left\|\left(x_{n}\right)_{t}(\theta)-\left(x_{0}\right)_{t}(\theta)\right\| d \theta \\
& =\int_{t-b}^{t}\left\|\left(x_{n}\right)_{t}(\theta)-\left(x_{0}\right)_{t}(\theta)\right\| d(t+\theta) \\
& =\int_{0}^{t}\left\|x_{n}(t+\theta)-x_{0}(t+\theta)\right\| d(t+\theta) \\
& =\int_{0}^{t}\left\|x_{n}(s)-x_{0}(s)\right\| d s .
\end{aligned}
$$

(ii) if $t \geq b$, then

$$
\begin{aligned}
\left\|\left(x_{n}\right)_{t}-\left(x_{0}\right)_{t}\right\|_{L[-b, 0]} & =\int_{-b}^{0}\left\|\left(x_{n}\right)_{t}(\theta)-\left(x_{0}\right)_{t}(\theta)\right\| d \theta \\
& =\int_{t-b}^{t}\left\|\left(x_{n}\right)_{t}(\theta)-\left(x_{0}\right)_{t}(\theta)\right\| d(t+\theta) \\
& =\int_{t-b}^{t}\left\|x_{n}(t+\theta)-x_{0}(t+\theta)\right\| d(t+\theta) \\
& =\int_{t-b}^{t}\left\|x_{n}(s)-x_{0}(s)\right\| d s .
\end{aligned}
$$

Obviously, (i) and (ii) imply that

$$
\left\|\left(x_{n}\right)_{t}-\left(x_{0}\right)_{t}\right\|_{L[-b, 0]} \leq b\left\|x_{n}-x_{0}\right\|_{P C(I, X)}, \forall t \in I .
$$

This completes the proof.

Lemma 5. Let $D=\left\{x_{n}\right\}_{n=1}^{\infty}$ be a bounded countable sequence in $P C(I, X)$. Then for each $t \in I$, one has

$$
\beta\left(D_{t}\right) \leq b \beta(D)
$$

where $D_{t}=\left\{\left(x_{n}\right)_{t}\right\}_{n=1}^{\infty}$.

Proof. From the definition of Kuratowski measures of noncompactness in Lemma 2, we can infer that for any $\varepsilon>0$, there is a partition $D=\bigcup_{i=1}^{k} D_{i}$ such that

$$
\operatorname{diam}\left(D_{i}\right)<\beta(D)+\varepsilon, i=1,2, \cdots, k
$$

As already done in Lemma 4, we also deduce

$$
\left\|\left(x_{n}\right)_{t}-\left(x_{m}\right)_{t}\right\|_{L[-b, 0]} \leq b\left\|x_{n}-x_{m}\right\|_{P C(I, X)}, t \in I .
$$

Hence, from (5) and (6) one derives

$$
\operatorname{diam}\left(D_{i_{t}}\right) \leq b \operatorname{diam}\left(D_{i}\right)<b(\beta(D)+\varepsilon), i=1,2, \cdots, k,
$$

which means

$$
\beta\left(D_{t}\right)<b \beta(D)+b \varepsilon .
$$

The arbitrariness of $\varepsilon$ implies that the conclusion is true. 


\section{Main Results}

In this section, we always suppose the resolvent operator $\{\wp(t)\}_{t \geq 0}$ for (4) to be differentiable. Based on [30], Definition 5 and the Riemann-Liouville standard fractional integral, the mild solution of system (1) can be defined as below.

Definition 6. For any given $u \in L^{2}(I, U)$, a function $x \in P C(J, X)$ is called a mild solution of system (1) on J, provided that $\int_{t_{k-1}}^{t_{k}}\left(t_{k}-s\right)^{\gamma-1} x(s) d s, \int_{t_{k}}^{t}(t-s)^{\gamma-1} x(s) d s \in \mathcal{D}$ for all $0<t_{k}<$ $t, t \in[0, \tau]$ and

$$
x(t)=\left\{\begin{aligned}
\phi(0) & +\frac{1}{\Gamma(\gamma)} A\left(\sum_{0<t_{k}<t} \int_{t_{k-1}}^{t_{k}}\left(t_{k}-s\right)^{\gamma-1} x(s) d s+\int_{t_{k}}^{t}(t-s)^{\gamma-1} x(s) d s\right) \\
& +\frac{1}{\Gamma(\gamma)}\left(\sum_{0<t_{k}<t} \int_{t_{k-1}}^{t_{k}}\left(t_{k}-s\right)^{\gamma-1}\left(f\left(s, x(s), x_{s}\right)+B u(s)\right) d s\right) \\
& +\frac{1}{\Gamma(\gamma)} \int_{t_{k}}^{t}(t-s)^{\gamma-1}\left(f\left(s, x(s), x_{s}\right)+B u(s)\right) d s+\sum_{0<t_{k}<t} I_{k}\left(x\left(t_{k}\right)\right), \quad t \in[0, \tau], \\
\phi(t), & t \in[-b, 0],
\end{aligned}\right.
$$

where $J=[-b, \tau], \tau \in(0, a]$.

Based on the exact controllability considered in [13], we give the following definition

Definition 7. System (1) is exact controllability on $I=[0, a]$ if for any initial function $\phi(t) \in$ $C([-b, 0], X)$ and $x_{1} \in X$, there has a control $u \in L^{2}(I, U)$ and a constant $\tau \in(0, a]$ such that the mild solution $x$ of $(1)$ on $J=[-b, \tau]$ satisfies $x(\tau)=x_{1}$.

Remark 2. In contrast with the existing definitions in [9-11,15,17], our target point $x_{1}$ taking value at $\tau \in(0, a]$ is likely to be achieved ahead of time $a$, which means that, from a conceptual point of view, it can be considered as an generalization of the existing notion of exact controllability.

In order to obtain the main results, we present the hypotheses as follows:

(H1) $f \in C(I \times X \times L([-b, 0], X), \mathcal{D})$ and satisfies

(i) $f$ maps bounded sets in $I \times X \times L([-b, 0], X)$ into bounded sets in $\mathcal{D}$;

(ii) There exist a constant $q \in(0, \gamma)$ and a function $l \in L^{\frac{1}{q}}\left(I, R^{+}\right)$such that for any bounded subsets $D_{1} \subset X, D_{2} \subset L([-b, 0], X)$,

$$
\beta\left(f\left(t, D_{1}, D_{2}\right)\right) \leq l(t)\left(\beta\left(D_{1}\right)+\beta\left(D_{2}\right)\right), t \in I .
$$

(H2) (i) The linear operator $B: L^{2}(I, U) \rightarrow L^{1}(I, \mathcal{D})$ is bounded, and there exists a constant $M_{1}>0$ satisfying $\|B\|_{\mathcal{L}(U, \mathcal{D})} \leq M_{1}$;

(ii) Linear operators $\mathfrak{J}(t), t \in I$, denoted by $\mathfrak{J}(\cdot)$ from $L^{2}(I, U)$ to $X$ defined as

$$
\begin{aligned}
\mathfrak{J}(t) u= & \frac{1}{\Gamma(\gamma)}\left(\sum_{0<t_{k}<t} \int_{t_{k-1}}^{t_{k}}\left(t_{k}-s\right)^{\gamma-1} B u(s) d s+\int_{t_{k}}^{t}(t-s)^{\gamma-1} B u(s) d s\right) \\
& +\frac{1}{\Gamma(\gamma)} \int_{0}^{t} \dot{\wp}(t-s)\left(\sum_{0<t_{k}<s} \int_{t_{k-1}}^{t_{k}}\left(t_{k}-\eta\right)^{\gamma-1} B u(\eta) d \eta+\int_{t_{k}}^{s}(s-\eta)^{\gamma-1} B u(\eta) d \eta\right) d s, t \in I,
\end{aligned}
$$


have invertible operators $\mathfrak{J}^{-1}(\cdot)$ taking values in $L^{2}(I, U) / \operatorname{ker} \mathfrak{J}(\cdot)$, which satisfy, for some constant $M_{2}>0$, sup $\left\|\mathfrak{J}^{-1}(\cdot)\right\|_{\mathcal{L}\left(X, L^{2}(I, U) / \text { ker } \mathfrak{J}(\cdot)\right)} \leq M_{2}$, and there is a constant $p \in(0, \gamma)$ and a function $k \in L^{\frac{1}{p}}\left(I, R^{+}\right)$satisfying

$$
\beta\left(\mathfrak{J}^{-1}(\cdot)(D)(s)\right) \leq k(s) \beta(D), s \in I,
$$

for any bounded subset $D \subset X$.

(H3) $I_{i}: X \rightarrow \mathcal{D}(i=1,2, \cdots, m)$ is continuous and satisfies

(i) There exists a constant $C_{0}$ such that

$$
\sup \left\{\left\|I_{i}(x)\right\|_{\mathcal{D}}, x \in X, i=1,2, \cdots, m\right\} \leq C_{0} ;
$$

(ii) There exist constants $k_{i} \geq 0$ such that

$$
\beta\left(I_{i}(D)\right) \leq k_{i} \beta(D), i=1,2, \cdots, m,
$$

hold for each bounded subset $D \subset X$.

(H4)

$$
\left(1+2\|\varphi\|_{L^{1}(I)}\right)(1+M) \sum_{i=1}^{m} k_{i}<1
$$

where

$$
M=\frac{1+2 \rho M_{1}\left(1+2\|\varphi\|_{L^{1}(I)}\right)}{\Gamma(\gamma)}, \rho=(m+1) a^{\gamma}\left(\frac{1-p}{\gamma-p}\right)^{1-p}\|k\|_{L^{\frac{1}{p}}},
$$

and $\varphi$ is the function mentioned in Definition 4 .

In the sequel, suppose $R_{0}$ to be a fixed constant such that $R_{0}>\left(\|\phi(0)\|_{\mathcal{D}}+m C_{0}\right)(1+$ $\left.\|\varphi\|_{L^{1}(I)}\right)$. By (H1), let

$$
M_{0}=\sup \left\{\|f(t, x, y)\|_{\mathcal{D}}:\|x\|_{P C(I, X)} \leq R_{0},\|y\|_{L[-b, 0]} \leq b\left(\|\phi\|_{b}+R_{0}\right), t \in I\right\} .
$$

For simplicity, take

$$
\begin{aligned}
\Theta(t ; x ; u)= & \frac{1}{\Gamma(\gamma)}\left(\sum_{0<t_{k}<t} \int_{t_{k-1}}^{t_{k}}\left(t_{k}-s\right)^{\gamma-1} f\left(s, x(s), x_{s}\right) d s+\int_{t_{k}}^{t}(t-s)^{\gamma-1} f\left(s, x(s), x_{s}\right) d s\right) \\
& +\frac{1}{\Gamma(\gamma)}\left(\sum_{0<t_{k}<t} \int_{t_{k-1}}^{t_{k}}\left(t_{k}-s\right)^{\gamma-1} B u(s) d s+\int_{t_{k}}^{t}(t-s)^{\gamma-1} B u(s) d s\right),
\end{aligned}
$$

set

$$
I(t ; x)=\sum_{0<t_{k}<t} I_{k}\left(x\left(t_{k}\right)\right)
$$

and present two notations as follows:

$$
\lambda=2(b+1)\left(\frac{1-q}{\gamma-q}\right)^{1-q}\|l\|_{L^{\frac{1}{q}}}, \mu=\frac{(m+1) a^{\gamma}}{\Gamma(\gamma+1)} .
$$

In view of condition (H2) and (H4), for any $x(\cdot) \in P C(I, X)$ and any $x_{1} \in X, t \in I$, define a control

$$
u_{x}(t):=\mathfrak{J}^{-1}(\tau)\left(x_{1}-\phi(0)-\Theta_{f}(\tau ; x)-I(\tau ; x)-\int_{0}^{\tau} \dot{\wp}(\tau-s)\left(\phi(0)+\Theta_{f}(s ; x)+I(s ; x)\right) d s\right)(t),
$$




$$
\begin{aligned}
& \tau=\min \left\{a,\left(\frac{\left(R_{0}-\left(\|\phi(0)\|_{\mathcal{D}}+m C_{0}\right)\left(1+\|\varphi\|_{L^{1}(I)}\right)\right) \Gamma(\gamma+1)}{(m+1)\left(M_{0}+M_{1} M_{3}\right)\left(1+\|\varphi\|_{L^{1}(I)}\right)}\right)^{\frac{1}{\gamma}},\left(\frac{\left(1-\left(1+2\|\varphi\|_{L^{1}(I)}\right)(1+M) \sum_{i=1}^{m} k_{i}\right)}{2\left(1+2\|\varphi\|_{L^{1}(I)}\right)(m+1) \lambda M}\right)^{\frac{1}{\gamma}}\right\}, \\
& M_{3}=M_{2}\left(\left(1+\|\varphi\|_{L^{1}(I)}\right)\left(\|\phi(0)\|_{\mathcal{D}}+\mu M_{0}+m C_{0}\right)+\left\|x_{1}\right\|\right) \\
& \Theta_{f}(s ; x)=\frac{1}{\Gamma(\gamma)}\left(\sum_{0<t_{k}<s} \int_{t_{k-1}}^{t_{k}}\left(t_{k}-\eta\right)^{\gamma-1} f\left(\eta, x(\eta), x_{\eta}\right) d \eta+\int_{t_{k}}^{s}(s-\eta)^{\gamma-1} f\left(\eta, x(\eta), x_{\eta}\right) d \eta\right), s \in[0, \tau] \text {. } \\
& \Omega=\left\{x \in P C(J, X):\|x\|_{P C([0, \tau], X)} \leq R_{0}, \sup _{t \in[0, \tau]}\left\|x_{t}\right\|_{L[-b, 0]} \leq b\left(\|\phi\|_{b}+R_{0}\right) ; x(t)=\phi(t), t \in[-b, 0]\right\} . \\
& (\mathcal{P} x)(t)=\left\{\begin{array}{l}
\phi(0)+\Theta\left(t ; x ; u_{x}\right)+I(t ; x) \\
+\int_{0}^{t} \dot{\wp}(t-s)\left(\phi(0)+\Theta\left(s ; x ; u_{x}\right)+I(s ; x)\right) d s, t \in[0, \tau], \\
\phi(t), t \in[-b, 0] .
\end{array}\right.
\end{aligned}
$$

To simplify the proof of our main result, the following lemmas are needed.

Lemma 6. Suppose that $f \in C(I \times X \times L([-b, 0], X), X)$ and $u \in L^{2}(I, U)$. Then $\Theta(\cdot ; x ; u) \in$ $C^{\gamma}\left(J_{i}, X\right), i=1,2, \cdots, m+1$, and

$$
[|\Theta(\cdot ; x ; u)|]_{C^{\gamma}} \leq \frac{2}{\gamma}\left(\|f\|_{C(I, X)}+\|B\|_{\mathcal{L}(U, X)} \cdot \sup _{t \in I}\|u(t)\|_{U}\right) .
$$

Proof. For $t \in J_{i}$ and $h>0$ such that $t+h \in J_{i}$, one has

$$
\begin{aligned}
& \|\Theta(t+h ; x ; u)-\Theta(t ; x ; u)\| \\
\leq & \int_{t_{i-1}}^{t}\left((t-s)^{\gamma-1}-(t+h-s)^{\gamma-1}\right)\left(\left\|f\left(s, x(s), x_{s}\right)\right\|+\|B u(s)\|\right) d s \\
& +\int_{t}^{t+h}(t+h-s)^{\gamma-1}\left(\left\|f\left(s, x(s), x_{s}\right)\right\|+\|B u(s)\|\right) d s \\
\leq & \left(\frac{\left(t-t_{i-1}\right)^{\gamma}-\left(t-t_{i-1}+h\right)^{\gamma}+h^{\gamma}}{\gamma}+\frac{h^{\gamma}}{\gamma}\right)\left(\|f\|_{C(I, X)}+\|B\|_{\mathcal{L}(U, X)} \cdot \sup _{t \in I}\|u(t)\|_{U}\right) \\
\leq & \frac{2 h^{\gamma}}{\gamma}\left(\|f\|_{C(I, X)}+\|B\|_{\mathcal{L}(U, X)} \cdot \sup _{t \in I}\|u(t)\|_{U}\right),
\end{aligned}
$$

which shows that $[|\Theta(\cdot ; x ; u)|]_{C \gamma} \leq \frac{2}{\gamma}\left(\|f\|_{C(I, X)}+\|B\|_{\mathcal{L}(U, X)} \cdot \sup _{t \in I}\|u(t)\|_{U}\right)$ and $\Theta(\cdot ; x ; u) \in$ $C^{\gamma}\left(J_{i}, X\right), i=1,2, \cdots, m+1$. This completes the proof.

Lemma 7. Assume that condition (H1) holds. Then the operator $T: P C(I, X) \rightarrow P C(I, X)$ defined by

$$
(T x)(t)=\int_{t^{*}}^{t}(t-s)^{\gamma-1} f\left(s, x(s), x_{s}\right) d s, \quad \forall t^{*}, t \in I,
$$

satisfies $\beta(T(D)(t)) \leq \lambda a^{\gamma} \beta(D)$ for any countable bounded set $D \subset P C(I, X)$. 
Proof. No loss of generality, we may suppose that the bounded countable set $D=\left\{x_{n}\right\}_{n=1}^{\infty}$. By using Lemma 5, we have

$$
\beta\left(\left\{\left(x_{n}\right)_{s}\right\}\right) \leq b \beta\left(\left\{x_{n}\right\}\right) .
$$

From Lemma 2 (II) and the Hölder inequality, it follows that

$$
\begin{aligned}
\beta(T(D)(t)) & =\beta\left(\left\{T\left(x_{n}\right)(t)\right\}\right) \\
& \leq 2 \int_{t^{*}}^{t}(t-s)^{\gamma-1} \beta\left(\left\{f\left(s, x_{n}(s),\left(x_{n}\right)_{s}\right)\right\}\right) d s \\
& \leq 2 \int_{t^{*}}^{t}(t-s)^{\gamma-1} l(s)\left(\beta\left(\left\{x_{n}\right\}\right)+\beta\left(\left\{\left(x_{n}\right)_{s}\right\}\right)\right) d s \\
& \leq 2(b+1) \int_{t^{*}}^{t}(t-s)^{\gamma-1} l(s) d s \cdot \beta\left(\left\{x_{n}\right\}\right) \\
& \leq 2(b+1)\left(\int_{t^{*}}^{t}\left[(t-s)^{\gamma-1}\right]^{\frac{1}{1-q}} d s\right)^{1-q}\left(\int_{t^{*}}^{t} l(s)^{\frac{1}{q}} d s\right)^{q} \cdot \beta\left(\left\{x_{n}\right\}\right) \\
& \leq 2(b+1)\left(\frac{1-q}{\gamma-q}\right)^{1-q}\left(t-t^{*}\right)^{\gamma-q}\|l\|_{L^{\frac{1}{q}}} \cdot \beta\left(\left\{x_{n}\right\}\right) \\
& \leq \lambda a^{\gamma} \beta(D) .
\end{aligned}
$$

This completes the proof.

Lemma 8. Assume that conditions (H1)(i), (H2), (H3)(i) and (H4) hold. Then $\{\mathcal{P} x: x \in \Omega\}$ is equicontinuous on each $J_{i}(i=0,1, \cdots, m+1)$.

Proof. The first step is to demonstrate that $\mathcal{P}(\Omega) \subseteq \Omega$. From (H2), one has

$$
\begin{aligned}
& \left\|u_{x}(t)\right\|_{U} \\
\leq & M_{2}\left(\left\|x_{1}\right\|+\|\phi(0)\|_{\mathcal{D}}+\sum_{0<t_{k}<\tau}\left\|I_{k}\left(x\left(t_{k}\right)\right)\right\|_{\mathcal{D}}+\int_{0}^{\tau} \varphi(\tau-s)\left(\|\phi(0)\|_{\mathcal{D}}+\sum_{0<t_{k}<s}\left\|I_{k}\left(x\left(t_{k}\right)\right)\right\|_{\mathcal{D}}\right) d s\right) \\
& +\frac{M_{2}}{\Gamma(\gamma)}\left(\sum_{0<t_{k}<\tau} \int_{t_{k-1}}^{t_{k}}\left(t_{k}-s\right)^{\gamma-1}\left\|f\left(s, x(s), x_{s}\right)\right\|_{\mathcal{D}} d s+\int_{t_{k}}^{\tau}(\tau-s)^{\gamma-1}\left\|f\left(s, x(s), x_{s}\right)\right\|_{\mathcal{D}} d s\right) \\
& +\frac{M_{2}}{\Gamma(\gamma)} \int_{0}^{\tau} \varphi(\tau-s)\left(\sum_{0<t_{k}<s} \int_{t_{k-1}}^{t_{k}}\left(t_{k}-\eta\right)^{\gamma-1}\left\|f\left(\eta, x(\eta), x_{\eta}\right)\right\|_{\mathcal{D}} d \eta+\int_{t_{k}}^{s}(s-\eta)^{\gamma-1}\left\|f\left(\eta, x(\eta), x_{\eta}\right)\right\|_{\mathcal{D}} d \eta\right) d s \\
\leq & M_{2}\left(\left\|x_{1}\right\|+\|\phi(0)\|_{\mathcal{D}}+m C_{0}+\|\varphi\|_{L^{1}(I)}\left(\|\phi(0)\|_{\mathcal{D}}+m C_{0}\right)\right)+M_{2} \frac{(m+1) M_{0} a^{\gamma}}{\Gamma(\gamma+1)}+M_{2}\|\varphi\|_{L^{1}(I)} \frac{(m+1) M_{0} a \gamma}{\Gamma(\gamma+1)} \\
\leq & M_{2}\left(\left(\|\varphi\|_{L^{1}(I)}+1\right)\left(\|\phi(0)\|_{\mathcal{D}}+\frac{(m+1) M_{0} a^{\gamma}}{\Gamma(\gamma+1)}+m C_{0}\right)+\left\|x_{1}\right\|\right)=M_{3}, t \in I .
\end{aligned}
$$

For any $x \in \Omega$ and $t \in[0, \tau]$, we obtain from (H2)

$$
\begin{aligned}
\left\|\Theta\left(t ; x ; u_{x}\right)\right\|_{\mathcal{D}} \leq & \frac{1}{\Gamma(\gamma)}\left(\sum_{0<t_{k}<t} \int_{t_{k-1}}^{t_{k}}\left(t_{k}-s\right)^{\gamma-1}\left\|f\left(s, x(s), x_{s}\right)\right\|_{\mathcal{D}} d s+\int_{t_{k}}^{t}(t-s)^{\gamma-1}\left\|f\left(s, x(s), x_{s}\right)\right\|_{\mathcal{D}} d s\right) \\
& +\frac{1}{\Gamma(\gamma)}\left(\sum_{0<t_{k}<t} \int_{t_{k-1}}^{t_{k}}\left(t_{k}-s\right)^{\gamma-1}\left\|B u_{x}(s)\right\|_{\mathcal{D}} d s+\int_{t_{k}}^{t}(t-s)^{\gamma-1}\left\|B u_{x}(s)\right\|_{\mathcal{D}} d s\right) \\
\leq & \frac{(m+1) M_{0} \tau^{\gamma}}{\Gamma(\gamma+1)}+\frac{(m+1) M_{1} M_{3} \tau^{\gamma}}{\Gamma(\gamma+1)}=\frac{(m+1)\left(M_{0}+M_{1} M_{3}\right) \tau^{\gamma}}{\Gamma(\gamma+1)} .
\end{aligned}
$$

Thus, this together with (7) shows 


$$
\begin{aligned}
\|(\mathcal{P} x)(t)\| \leq & \|\phi(0)\|_{\mathcal{D}}+\left\|\Theta\left(t ; x ; u_{x}\right)\right\|_{\mathcal{D}}+\|I(t ; x)\|_{\mathcal{D}} \\
& +\int_{0}^{t} \varphi(t-s)\left(\|\phi(0)\|_{\mathcal{D}}+\left\|\Theta\left(s ; x ; u_{x}\right)\right\|_{\mathcal{D}}+\|I(s ; x)\|_{\mathcal{D}}\right) d s \\
\leq & \|\phi(0)\|_{\mathcal{D}}+\frac{(m+1)\left(M_{0}+M_{1} M_{3}\right) \tau^{\gamma}}{\Gamma(\gamma+1)}+m C_{0} \\
& +\|\varphi\|_{L^{1}(I)}\left(\|\phi(0)\|_{\mathcal{D}}+\frac{(m+1)\left(M_{0}+M_{1} M_{3}\right) \tau^{\gamma}}{\Gamma(\gamma+1)}+m C_{0}\right) \\
\leq & \left(\|\phi(0)\|_{\mathcal{D}}+m C_{0}\right)\left(\|\varphi\|_{L^{1}(I)}+1\right)+\frac{(m+1)\left(M_{0}+M_{1} M_{3}\right)\left(\|\varphi\|_{L^{1}(I)}+1\right)}{\Gamma(\gamma+1)} \tau^{\gamma} \\
\leq & R_{0} . \\
& \text { On the other hand, } \\
& \quad\left\|(\mathcal{P} x)_{t}\right\|_{L[-b, 0]}=\int_{-b}^{0}\left\|(\mathcal{P} x)_{t}(\theta)\right\| d \theta= \begin{cases}\int_{t-b}^{0}\|\phi(s)\| d s+\int_{0}^{t}\|(\mathcal{P} x)(s)\| d s, & t \leq b, \\
\int_{t-b}^{t}\|(\mathcal{P} x)(s)\| d s, & t \geq b,\end{cases}
\end{aligned}
$$

which means

$$
\left\|(\mathcal{P} x)_{t}\right\|_{L[-b, 0]} \leq b\|\phi\|_{b}+b\|\mathcal{P} x\|_{P C([0, t], X)} .
$$

Then, we have

$$
\sup _{t \in[0, \tau]}\left\|(\mathcal{P} x)_{t}\right\|_{L[-b, 0]} \leq b\left(\|\phi\|_{b}+R_{0}\right) .
$$

It is obvious that $(\mathcal{P} x)(t)=\phi(t)$ for any $t \in[-b, 0]$. Then the fact $\mathcal{P}(\Omega) \subseteq \Omega$ is thus proved.

Next, we shall prove that $\{\mathcal{P} x: x \in \Omega\}$ is equicontinuous on each $J_{i}$. For any $x \in \Omega$ and $\xi_{1}, \xi_{2} \in J_{i}$ with $\xi_{1}<\xi_{2}$, the discussion can be divided into two cases.

Case (i): If $\xi_{1}, \xi_{2} \in J_{0}$, then from the continuity of $\phi(\cdot)$, we have

$$
\left\|(\mathcal{P} x)\left(\xi_{2}\right)-(\mathcal{P} x)\left(\xi_{1}\right)\right\|=\left\|\phi\left(\xi_{2}\right)-\phi\left(\xi_{1}\right)\right\| \rightarrow 0 \text {, as }\left|\xi_{1}-\xi_{2}\right| \rightarrow 0 .
$$

Case (ii): If $\xi_{1}, \xi_{2} \in J_{i}, i>0$, then

$$
\begin{aligned}
& (\mathcal{P} x)\left(\xi_{2}\right)-(\mathcal{P} x)\left(\xi_{1}\right) \\
= & \Theta\left(\xi_{2} ; x ; u_{x}\right)-\Theta\left(\xi_{1} ; x ; u_{x}\right)+I\left(\xi_{2} ; x\right)-I\left(\xi_{1} ; x\right) \\
& +\int_{0}^{\xi_{2}} \dot{\wp}\left(\xi_{2}-s\right) \phi(0) d s-\int_{0}^{\xi_{1}} \dot{\wp}\left(\xi_{1}-s\right) \phi(0) d s \\
& +\int_{0}^{\xi_{2}} \dot{\wp}\left(\xi_{2}-s\right) \Theta\left(s ; x ; u_{x}\right) d s-\int_{0}^{\xi_{1}} \dot{\wp}\left(\xi_{1}-s\right) \Theta\left(s ; x ; u_{x}\right) d s \\
& +\int_{0}^{\xi_{2}} \dot{\wp}\left(\xi_{2}-s\right) I(s ; x) d s-\int_{0}^{\xi_{1}} \dot{\wp}\left(\xi_{1}-s\right) I(s ; x) d s .
\end{aligned}
$$

Denote by

$$
\begin{aligned}
& \Lambda_{1}=\Theta\left(\xi_{2} ; x ; u_{x}\right)-\Theta\left(\xi_{1} ; x ; u_{x}\right)+I\left(\xi_{2} ; x\right)-I\left(\xi_{1} ; x\right) \\
& \Lambda_{2}=\int_{0}^{\xi_{2}} \dot{\wp}\left(\xi_{2}-s\right) \phi(0) d s-\int_{0}^{\xi_{1}} \dot{\wp}\left(\xi_{1}-s\right) \phi(0) d s ; \\
& \Lambda_{3}=\int_{0}^{\xi_{2}} \dot{\wp}\left(\xi_{2}-s\right)\left(\Theta\left(s ; x ; u_{x}\right)+I(s ; x)\right) d s-\int_{0}^{\xi_{1}} \dot{\wp}\left(\xi_{1}-s\right)\left(\Theta\left(s ; x ; u_{x}\right)+I(s ; x)\right) d s .
\end{aligned}
$$

Then we have

$$
\left\|(\mathcal{P} x)\left(\xi_{2}\right)-(\mathcal{P} x)\left(\xi_{1}\right)\right\| \leq\left\|\Lambda_{1}\right\|+\left\|\Lambda_{2}\right\|+\left\|\Lambda_{3}\right\| .
$$

In the following, we prove that $\left\|\Lambda_{i}\right\| \rightarrow 0$ independently of $x \in \Omega$ as $\left|\xi_{1}-\xi_{2}\right| \rightarrow$ $0, i=1,2,3$. For $\Lambda_{1}$, we have 


$$
\begin{aligned}
\left\|\Lambda_{1}\right\| \leq & \left\|\int_{t_{i-1}}^{\xi_{2}}\left(\xi_{2}-s\right)^{\gamma-1} f\left(s, x(s), x_{s}\right) d s-\int_{t_{i-1}}^{\xi_{1}}\left(\xi_{1}-s\right)^{\gamma-1} f\left(s, x(s), x_{s}\right) d s\right\| \\
& +\left\|\int_{t_{i-1}}^{\xi_{2}}\left(\xi_{2}-s\right)^{\gamma-1} B u_{x}(s) d s-\int_{t_{i-1}}^{\xi_{1}}\left(\xi_{1}-s\right)^{\gamma-1} B u_{x}(s) d s\right\| \\
\leq & \int_{t_{i-1}}^{\xi_{1}}\left[\left(\xi_{1}-s\right)^{\gamma-1}-\left(\xi_{2}-s\right)^{\gamma-1}\right]\left\|f\left(s, x(s), x_{s}\right)\right\|_{\mathcal{D}} d s+\int_{\xi_{1}}^{\xi_{2}}\left(\xi_{2}-s\right)^{\gamma-1}\left\|f\left(s, x(s), x_{s}\right)\right\|_{\mathcal{D}} d s \\
& +\int_{t_{i-1}}^{\xi_{1}}\left[\left(\xi_{1}-s\right)^{\gamma-1}-\left(\xi_{2}-s\right)^{\gamma-1}\right]\left\|B u_{x}(s)\right\|_{\mathcal{D}} d s+\int_{\xi_{1}}^{\xi_{2}}\left(\xi_{2}-s\right)^{\gamma-1}\left\|B u_{x}(s)\right\|_{\mathcal{D}} d s \\
\leq & \frac{M_{0}}{\gamma}\left[\left(\xi_{1}-t_{i-1}\right)^{\gamma}-\left(\xi_{2}-t_{i-1}\right)^{\gamma}+\left(\xi_{2}-\xi_{1}\right)^{\gamma}\right]+\frac{M_{0}}{\gamma}\left(\xi_{2}-\xi_{1}\right)^{\gamma} \\
& +\frac{M_{1} M_{3}}{\gamma}\left[\left(\xi_{1}-t_{i-1}\right)^{\gamma}-\left(\xi_{2}-t_{i-1}\right)^{\gamma}+\left(\xi_{2}-\xi_{1}\right)^{\gamma}\right]+\frac{M_{1} M_{3}}{\gamma}\left(\xi_{2}-\xi_{1}\right)^{\gamma} \\
& \rightarrow 0, \text { as }\left|\xi_{1}-\xi_{2}\right| \rightarrow 0 .
\end{aligned}
$$

For $\Lambda_{2}$, we can rewrite it as

$$
\begin{aligned}
\Lambda_{2} & =\int_{0}^{\xi_{2}} \dot{\wp}\left(\xi_{2}-s\right) \phi(0) d s-\int_{0}^{\xi_{1}} \dot{\wp}\left(\xi_{1}-s\right) \phi(0) d s \\
& =\int_{0}^{\xi_{2}-\xi_{1}} \dot{\wp}\left(\xi_{2}-s\right) \phi(0) d s+\int_{\tilde{\xi}_{2}-\xi_{1}}^{\xi_{2}} \dot{\wp}\left(\xi_{2}-s\right) \phi(0) d s-\int_{0}^{\xi_{1}} \dot{\wp}\left(\xi_{1}-s\right) \phi(0) d s \\
& =\int_{0}^{\xi_{2}-\xi_{1}} \dot{\wp}\left(\xi_{2}-s\right) \phi(0) d s .
\end{aligned}
$$

By Definition 4, one gets

$$
\left\|\Lambda_{2}\right\| \leq\|\phi(0)\|_{\mathcal{D}} \int_{0}^{\xi_{2}-\xi_{1}} \varphi\left(\xi_{2}-s\right) d s \rightarrow 0, \text { as }\left|\xi_{1}-\xi_{2}\right| \rightarrow 0 .
$$

From the proof process of Lemma 6 and (9), it follows that

$$
\begin{aligned}
\left\|\Lambda_{3}\right\|= & \left\|\int_{0}^{\xi_{2}} \dot{\wp}\left(\xi_{2}-s\right)\left(\Theta\left(s ; x ; u_{x}\right)+I(s ; x)\right) d s-\int_{0}^{\xi_{1}} \dot{\wp}\left(\xi_{1}-s\right)\left(\Theta\left(s ; x ; u_{x}\right)+I(s ; x)\right) d s\right\| \\
\leq & \left\|\int_{0}^{\xi_{2}-\xi_{1}} \dot{\wp}\left(\xi_{2}-s\right) \Theta\left(s ; x ; u_{x}\right) d s+\int_{\xi_{2}-\xi_{1}}^{\xi_{2}} \dot{\wp}\left(\xi_{2}-s\right) \Theta\left(s ; x ; u_{x}\right) d s-\int_{0}^{\xi_{1}} \dot{\wp}\left(\xi_{1}-s\right) \Theta\left(s ; x ; u_{x}\right) d s\right\| \\
& +\left\|\int_{0}^{\xi_{2}} \dot{\wp}\left(\xi_{2}-s\right) I(s ; x) d s-\int_{0}^{\xi_{1}} \dot{\wp}\left(\xi_{1}-s\right) I(s ; x) d s\right\| \\
\leq & \left\|\int_{0}^{\xi_{2}-\xi_{1}} \dot{\wp}\left(\xi_{2}-s\right) \Theta\left(s ; x ; u_{x}\right) d s+\int_{0}^{\xi_{1}} \dot{\wp}(s) \Theta\left(\xi_{2}-s ; x ; u_{x}\right) d s-\int_{0}^{\xi_{1}} \dot{\wp}(s) \Theta\left(\xi_{1}-s ; x ; u_{x}\right) d s\right\| \\
& +\left\|\int_{0}^{\xi_{2}-\xi_{1}} \dot{\wp}\left(\xi_{2}-s\right) I(s ; x) d s+\int_{0}^{\xi_{1}} \dot{\wp}(s)\left[I\left(\xi_{2}-s ; x\right)-I\left(\xi_{1}-s ; x\right)\right] d s\right\| \\
\leq & \int_{0}^{\xi_{2}-\xi_{1}} \varphi\left(\xi_{2}-s\right)\left\|\Theta\left(s ; x ; u_{x}\right)\right\|_{\mathcal{D}} d s+\int_{0}^{\xi_{1}} \varphi(s)\left\|\Theta\left(\xi_{2}-s ; x ; u_{x}\right)-\Theta\left(\xi_{1}-s ; x ; u_{x}\right)\right\|_{\mathcal{D}} d s \\
& +\int_{0}^{\xi_{2}-\xi_{1}} \varphi\left(\xi_{2}-s\right)\|I(s ; x)\|_{\mathcal{D}} d s+\int_{0}^{\xi_{1}} \varphi(s)\left\|I\left(\xi_{2}-s ; x\right)-I\left(\xi_{1}-s ; x\right)\right\|_{\mathcal{D}} d s \\
\leq & \int_{0}^{\xi_{2}-\xi_{1}} \varphi\left(\xi_{2}-s\right) d s \cdot \frac{(m+1)\left(M_{0}+M_{1} M_{3}\right) \tau^{\gamma}}{\Gamma(\gamma+1)}+\int_{0}^{\xi_{1}} \varphi(s) d s \cdot \frac{2\left(M_{0}+M_{1} M_{3}\right)}{\gamma}\left(\xi_{2}-\xi_{1}\right)^{\gamma} \\
& +m C_{0} \int_{0}^{\xi_{2}-\xi_{1}} \varphi\left(\xi_{2}-s\right) d s \\
& \rightarrow 0, a s\left|\xi_{1}-\xi_{2}\right| \rightarrow 0 .
\end{aligned}
$$

To sum up, it can be concluded that $\left\|(\mathcal{P} x)\left(\xi_{2}\right)-(\mathcal{P} x)\left(\xi_{1}\right)\right\| \rightarrow 0$, as $\left|\xi_{1}-\xi_{2}\right| \rightarrow 0$, for all $x \in \Omega$. Consequently, $\{\mathcal{P} x: x \in \Omega\}$ is equicontinuous on each $J_{i}(i=0,1, \cdots, m+1)$.

Lemma 9. Assume that conditions (H1)(i), (H2), (H3)(i) and (H4) hold. Then the operator $\mathcal{P}: \Omega \rightarrow \Omega$ is continuous.

Proof. Since $\mathcal{P}(\Omega) \subseteq \Omega$ from Lemma 8 , we only need to prove that $\mathcal{P}$ is continuous. Suppose $\left\{y_{n}\right\}$ to be a sequence satisfying $y_{n} \rightarrow y$ in $\Omega$ as $n \rightarrow \infty$. 
From condition (H3), it is easy to see that

$$
\sum_{0<t_{k}<t}\left\|I_{k}\left(y_{n}\left(t_{k}\right)\right)-I_{k}\left(y\left(t_{k}\right)\right)\right\|_{\mathcal{D}} \rightarrow 0, t \in[0, \tau], \text { as } n \rightarrow+\infty .
$$

From condition (H1) and Lebesgue dominated convergence theorem, it follows that

$$
\int_{t^{*}}^{t}(t-s)^{\gamma-1}\left\|f\left(s, y_{n}(s),\left(y_{n}\right)_{s}\right)-f\left(s, y(s), y_{s}\right)\right\|_{\mathcal{D}} d s \rightarrow 0, \forall t^{*}, t \in[0, \tau], \text { as } n \rightarrow+\infty .
$$

Therefore, one can obtain

$$
\begin{aligned}
& \left\|B u_{y_{n}}(s)-B u_{y}(s)\right\|_{\mathcal{D}} \\
\leq & M_{1} M_{2}\left[\left\|\Theta_{f}\left(\tau ; y_{n}\right)-\Theta_{f}(\tau ; y)\right\|_{\mathcal{D}}+\left\|I\left(\tau ; y_{n}\right)-I(\tau ; y)\right\|_{\mathcal{D}}\right. \\
& \left.+\int_{0}^{\tau} \varphi(\tau-s)\left(\left\|\Theta_{f}\left(s ; y_{n}\right)-\Theta_{f}(s ; y)\right\|_{\mathcal{D}}+\left\|I\left(s ; y_{n}\right)-I(s ; y)\right\|_{\mathcal{D}}\right) d s\right] \\
\leq & M_{1} M_{2}\left[\sum_{0<t_{k}<\tau} \int_{t_{k-1}}^{t_{k}}\left(t_{k}-s\right)^{\gamma-1}\left\|f\left(s, y_{n}(s),\left(y_{n}\right)_{s}\right)-f\left(s, y(s), y_{s}\right)\right\|_{\mathcal{D}} d s\right. \\
& +\int_{t_{k}}^{\tau}(\tau-s)^{\gamma-1}\left\|f\left(s, y_{n}(s),\left(y_{n}\right)_{s}\right)-f\left(s, y(s), y_{s}\right)\right\|_{\mathcal{D}} d s+\sum_{0<t_{k}<\tau}\left\|I_{k}\left(y_{n}\left(t_{k}\right)\right)-I_{k}\left(y\left(t_{k}\right)\right)\right\|_{D} \\
& +\int_{0}^{\tau} \varphi(\tau-s)\left(\sum_{0<t_{k}<s} \int_{t_{k-1}}^{t_{k}}\left(t_{k}-\eta\right)^{\gamma-1}\left\|f\left(\eta, y_{n}(\eta),\left(y_{n}\right)_{\eta}\right)-f\left(\eta, y(\eta), y_{\eta}\right)\right\|_{\mathcal{D}} d \eta\right. \\
& \left.\left.+\int_{t_{k}}^{s}(s-\eta)^{\gamma-1}\left\|f\left(\eta, y_{n}(\eta),\left(y_{n}\right)_{\eta}\right)-f\left(\eta, y(\eta), y_{\eta}\right)\right\|_{\mathcal{D}} d \eta+\sum_{0<t_{k}<s}\left\|I_{k}\left(y_{n}\left(t_{k}\right)\right)-I_{k}\left(y\left(t_{k}\right)\right)\right\|_{\mathcal{D}}\right) d s\right] \\
& \rightarrow 0, \text { as } n \rightarrow+\infty .
\end{aligned}
$$

Then, for each $t \in[0, \tau]$, one has

$$
\begin{aligned}
& \left\|\left(\mathcal{P} y_{n}\right)(t)-(\mathcal{P} y)(t)\right\| \\
\leq & \left\|\Theta\left(t ; y_{n} ; u_{y_{n}}\right)-\Theta\left(t ; y ; u_{y}\right)\right\|_{\mathcal{D}}+\left\|I\left(t ; y_{n}\right)-I(t ; y)\right\|_{\mathcal{D}} \\
& +\int_{0}^{t}\left\|\dot{\wp}(t-s)\left[\Theta\left(s ; y_{n} ; u_{y_{n}}\right)-\Theta\left(s ; y ; u_{y}\right)\right]\right\| d s+\int_{0}^{t}\left\|\dot{\wp}(t-s)\left[I\left(s ; y_{n}\right)-I(s ; y)\right]\right\| d s \\
\leq & \sum_{0<t_{k}<t} \int_{t_{k-1}}^{t_{k}}\left(t_{k}-s\right)^{\gamma-1}\left\|f\left(s, y_{n}(s),\left(y_{n}\right)_{s}\right)-f\left(s, y(s), y_{s}\right)\right\|_{\mathcal{D}} d s \\
& +\int_{t_{k}}^{t}(t-s)^{\gamma-1}\left\|f\left(s, y_{n}(s),\left(y_{n}\right)_{s}\right)-f\left(s, y(s), y_{s}\right)\right\|_{\mathcal{D}} d s+\sum_{0<t_{k}<t}\left\|I_{k}\left(y_{n}\left(t_{k}\right)\right)-I_{k}\left(y\left(t_{k}\right)\right)\right\|_{\mathcal{D}} \\
& +\sum_{0<t_{k}<t} \int_{t_{k-1}}^{t_{k}}\left(t_{k}-s\right)^{\gamma-1}\left\|B u_{y_{n}}(s)-B u_{y}(s)\right\|_{\mathcal{D}} d s+\int_{t_{k}}^{t}(t-s)^{\gamma-1}\left\|B u_{y_{n}}(s)-B u_{y}(s)\right\|_{\mathcal{D}} d s \\
& +\int_{0}^{t} \varphi(t-s)\left(\sum_{0<t_{k}<s} \int_{t_{k-1}}^{t_{k}}\left(t_{k}-\eta\right)^{\gamma-1}\left\|f\left(\eta, y_{n}(\eta),\left(y_{n}\right)_{\eta}\right)-f\left(\eta, y(\eta), y_{\eta}\right)\right\|_{\mathcal{D}} d \eta\right. \\
& \left.+\int_{t_{k}}^{s}(s-\eta)^{\gamma-1}\left\|f\left(\eta, y_{n}(\eta),\left(y_{n}\right)_{\eta}\right)-f\left(\eta, y(\eta), y_{\eta}\right)\right\|_{\mathcal{D}} d \eta\right) d s \\
& +\int_{0}^{t} \varphi(t-s)\left(\sum_{0<t_{k}<s} \int_{t_{k-1}}^{t_{k}}\left(t_{k}-\eta\right)^{\gamma-1}\left\|B u_{y_{n}}(\eta)-B u_{y}(\eta)\right\|_{\mathcal{D}} d \eta\right. \\
& \left.+\int_{t_{k}}^{s}(s-\eta)^{\gamma-1}\left\|B u_{y_{n}}(\eta)-B u_{y}(\eta)\right\|_{\mathcal{D}} d \eta\right) d s+\int_{0}^{t} \varphi(t-s)\left(\sum_{0<t_{k}<s}\left\|I_{k}\left(y_{n}\left(t_{k}\right)\right)-I_{k}\left(y\left(t_{k}\right)\right)\right\|_{\mathcal{D}}\right) d s \\
& \rightarrow 0, a s n \rightarrow+\infty .
\end{aligned}
$$

By means of the similar proof of equicontinuous for $\{\mathcal{P} x: x \in \Omega\}$ in Lemma 8 and the Ascoli-Arzelà theorem, it is easy to get $\left\|\mathcal{P} y_{n}-\mathcal{P} y\right\|_{P C(J, X)} \rightarrow 0$, as $n \rightarrow+\infty$, i.e., $\mathcal{P}$ is continuous on $\Omega$. The conclusion follows.

Now, it is in the position to present our main theorem of this work. 
Theorem 1. Assume that hypotheses (H1)-(H4) hold, then the fractional evolution Equations (1) satisfies exact controllability on $I$.

Proof. From (8) and Lemma 1, we know that it is suffices to show that under control $u_{x}$ the operator $\mathcal{P}$ has a fixed point $x$ which is a mild solution of (1) on $J$. Simple verification implies the fact $x(\tau)=(\mathcal{P} x)(\tau)=x_{1}$ which can show that system (1) is exactly controllable on $I$. For this purpose, we shall take advantage of Mönch fixed point theorem.

The continuity of operator $\mathcal{P}: \Omega \rightarrow \Omega$ is given by Lemma 9. Take $B=\overline{c o} \mathcal{P}(\Omega)$. It is not difficult to check that $\mathcal{P}(B) \subseteq B$. Suppose $D_{0} \subset B$ to be a bounded countable set satisfying $D_{0} \subset \overline{c o}\left(\left\{x_{0}\right\} \cup \mathcal{P}\left(D_{0}\right)\right)$, we shall prove that $\beta\left(D_{0}\right)=0$. From Lemma 8 , it is easy to derive that $\mathcal{P}\left(D_{0}\right)$ is equicontinuous on $J_{i}, i=0,1, \cdots, m+1$. Notice that $D_{0} \subset \overline{c o}\left(\left\{x_{0}\right\} \cup \mathcal{P}\left(D_{0}\right)\right)$, so $D_{0}$ is also equicontinuous on each $J_{i}$.

For any $x \in D_{0}$, denote

$$
(\mathcal{P} x)(t)=\left\{\begin{array}{l}
\left(\mathcal{P}_{1} x\right)(t)+\left(\mathcal{P}_{2} x\right)(t)+\left(\mathcal{P}_{3} x\right)(t)+\left(\mathcal{P}_{4} x\right)(t), t \in[0, \tau], \\
\phi(t), t \in[-b, 0]
\end{array}\right.
$$

where

$$
\begin{aligned}
& \left(\mathcal{P}_{1} x\right)(t)=\phi(0)+I(t ; x) \\
& \left(\mathcal{P}_{2} x\right)(t)=\Theta\left(t ; x ; u_{x}\right) \\
& \left(\mathcal{P}_{3} x\right)(t)=\int_{0}^{t} \dot{\wp}(t-s) \Theta\left(s ; x ; u_{x}\right) d s \\
& \left(\mathcal{P}_{4} x\right)(t)=\int_{0}^{t} \dot{\wp}(t-s)(\phi(0)+I(s ; x)) d s .
\end{aligned}
$$

Without loss of generality, let $D_{0}=\left\{z_{n}\right\}_{n=1}^{\infty}$. Then it is not difficult to obtain that

$$
\begin{aligned}
\beta\left(\mathcal{P}_{1}\left(D_{0}\right)(t)\right) & =\beta\left(\left\{\phi(0)+\sum_{0<t_{k}<t} I_{k}\left(z_{n}\left(t_{k}\right)\right)\right\}\right) \\
& \leq \sum_{i=1}^{m} k_{i} \beta\left(\left\{z_{n}\right\}\right) \\
& =\sum_{i=1}^{m} k_{i} \cdot \beta\left(D_{0}\right), t \in[0, \tau] .
\end{aligned}
$$

From hypothesis (H1)(ii) and Lemma 5, for any $s \in I$, we get

$$
\begin{aligned}
\beta\left(\left\{f\left(s, z_{n}(s),\left(z_{n}\right)_{s}\right)\right\}\right) & \leq l(s)\left(\beta\left(\left\{z_{n}(s)\right\}\right)+\beta\left(\left\{\left(z_{n}\right)_{s}\right\}\right)\right) \\
& \leq l(s)\left(\beta\left(\left\{z_{n}(s)\right\}\right)+b \beta\left(\left\{z_{n}\right\}\right)\right) \\
& \leq l(s)\left(\beta\left(D_{0}(s)\right)+b \beta\left(D_{0}\right)\right) \\
& \leq l(s)(b+1) \beta\left(D_{0}\right) .
\end{aligned}
$$

Then this implies from Lemma 2 and Lemma 7 that

$$
\begin{aligned}
& \beta\left(\left\{\Theta_{f}\left(t ; z_{n}\right)\right\}\right) \\
= & \beta\left(\left\{\frac{1}{\Gamma(\gamma)}\left(\sum_{0<t_{k}<t} \int_{t_{k-1}}^{t_{k}}\left(t_{k}-s\right)^{\gamma-1} f\left(s, z_{n}(s),\left(z_{n}\right)_{s}\right) d s+\int_{t_{k}}^{t}(t-s)^{\gamma-1} f\left(s, z_{n}(s),\left(z_{n}\right)_{s}\right) d s\right)\right\}\right) \\
\leq & \frac{2}{\Gamma(\gamma)}\left(\sum_{0<t_{k}<t} \int_{t_{k-1}}^{t_{k}}\left(t_{k}-s\right)^{\gamma-1} \beta\left(\left\{f\left(s, z_{n}(s),\left(z_{n}\right)_{s}\right)\right\}\right) d s+\int_{t_{k}}^{t}(t-s)^{\gamma-1} \beta\left(\left\{f\left(s, z_{n}(s),\left(z_{n}\right)_{s}\right)\right\}\right) d s\right) \\
\leq & \frac{2(m+1)}{\Gamma(\gamma)} \lambda \tau^{\gamma} \beta\left(D_{0}\right), t \in[0, \tau],
\end{aligned}
$$

which together with (H2) (ii) indicates 


$$
\begin{aligned}
& \beta\left(\left\{u_{z_{n}}(s)\right\}\right) \\
\leq & k(s)\left(\beta\left(\left\{\left(\mathcal{P}_{1} z_{n}\right)(\tau)\right\}\right)+\beta\left(\left\{\Theta_{f}\left(\tau ; z_{n}\right)\right\}\right)+2 \int_{0}^{\tau} \beta\left(\left\{\dot{\wp}(\tau-s)\left(\left(\mathcal{P}_{1} z_{n}\right)(s)+\Theta_{f}\left(s ; z_{n}\right)\right)\right\}\right) d s\right) \\
\leq & k(s)\left(\beta\left(\mathcal{P}_{1}\left(D_{0}\right)(\tau)\right)+\beta\left(\left\{\Theta_{f}\left(\tau ; z_{n}\right)\right\}\right)+2 \int_{0}^{\tau} \varphi(\tau-s)\left(\beta\left(\mathcal{P}_{1}\left(D_{0}\right)(s)\right)+\beta\left(\left\{\Theta_{f}\left(s ; z_{n}\right)\right\}\right)\right) d s\right) \\
\leq & k(s)\left(\left(\sum_{i=1}^{m} k_{i}+\frac{2(m+1)}{\Gamma(\gamma)} \lambda \tau^{\gamma}\right) \beta\left(D_{0}\right)+2\|\varphi\|_{L^{1}(I)}\left(\sum_{i=1}^{m} k_{i}+\frac{2(m+1)}{\Gamma(\gamma)} \lambda \tau^{\gamma}\right) \beta\left(D_{0}\right)\right) \\
\leq & k(s)\left(\sum_{i=1}^{m} k_{i}+\frac{2(m+1)}{\Gamma(\gamma)} \lambda \tau^{\gamma}\right)\left(1+2\|\varphi\|_{L^{1}(I)}\right) \beta\left(D_{0}\right), s \in[0, \tau] .
\end{aligned}
$$

In addition, by using Hölder inequality, we have

$$
\begin{aligned}
& \sum_{0<t_{k}<t} \int_{t_{k-1}}^{t_{k}}\left(t_{k}-s\right)^{\gamma-1} k(s) d s+\int_{t_{k}}^{t}(t-s)^{\gamma-1} k(s) d s \\
\leq & m\left(\frac{1-p}{\gamma-p}\right)^{1-p}\|k\|_{L^{\frac{1}{p}}} a^{\gamma}+\left(\frac{1-p}{\gamma-p}\right)^{1-p}\|k\|_{L^{\frac{1}{p}}} a^{\gamma} \\
= & \rho .
\end{aligned}
$$

Consequently,

$$
\begin{aligned}
& \beta\left(\mathcal{P}_{2}\left(D_{0}\right)(t)\right) \\
\leq & \beta\left(\left\{\Theta_{f}\left(t ; z_{n}\right)\right\}\right)+\beta\left(\left\{\frac{1}{\Gamma(\gamma)}\left(\sum_{0<t_{k}<t} \int_{t_{k-1}}^{t_{k}}\left(t_{k}-s\right)^{\gamma-1} B u_{z_{n}}(s) d s+\int_{t_{k}}^{t}(t-s)^{\gamma-1} B u_{z_{n}}(s) d s\right)\right\}\right) \\
\leq & \beta\left(\left\{\Theta_{f}\left(t ; z_{n}\right)\right\}\right)+\frac{2 M_{1}}{\Gamma(\gamma)}\left(\sum_{0<t_{k}<t} \int_{t_{k-1}}^{t_{k}}\left(t_{k}-s\right)^{\gamma-1} \beta\left(\left\{u_{z_{n}}(s)\right\}\right) d s+\int_{t_{k}}^{t}(t-s)^{\gamma-1} \beta\left(\left\{u_{z_{n}}(s)\right\}\right) d s\right) \\
\leq & \beta\left(\left\{\Theta_{f}\left(t ; z_{n}\right)\right\}\right) \\
& +\frac{2 M_{1}}{\Gamma(\gamma)}\left(\sum_{i=1}^{m} k_{i}+\frac{2(m+1)}{\Gamma(\gamma)} \lambda \tau^{\gamma}\right)\left(1+2\|\varphi\|_{L^{1}(I)}\right)\left(\sum_{0<t_{k}<t} \int_{t_{k-1}}^{t_{k}}\left(t_{k}-s\right)^{\gamma-1} k(s) d s+\int_{t_{k}}^{t}(t-s)^{\gamma-1} k(s) d s\right) \beta\left(D_{0}\right) \\
\leq & \frac{2(m+1)}{\Gamma(\gamma)} \lambda \tau^{\gamma} \beta\left(D_{0}\right)+\frac{2 \rho M_{1}}{\Gamma(\gamma)}\left(\sum_{i=1}^{m} k_{i}+\frac{2(m+1)}{\Gamma(\gamma)} \lambda \tau^{\gamma}\right)\left(1+2\|\varphi\|_{L^{1}(I)}\right) \beta\left(D_{0}\right) \\
\leq & \frac{1+2 \rho M_{1}\left(1+2\|\varphi\|_{L^{1}(I)}\right)}{\Gamma(\gamma)}\left(2(m+1) \lambda \tau^{\gamma}+\sum_{i=1}^{m} k_{i}\right) \beta\left(D_{0}\right) \\
= & \left(2(m+1) M \lambda \tau^{\gamma}+M \sum_{i=1}^{m} k_{i}\right) \beta\left(D_{0}\right) .
\end{aligned}
$$

From Lemma 2, for $t \in[0, \tau]$, we have

$$
\begin{aligned}
\beta\left(\mathcal{P}_{3}\left(D_{0}\right)(t)\right) & =\beta\left(\left\{\int_{0}^{t} \dot{\wp}(t-s) \Theta\left(s ; z_{n} ; u_{z_{n_{x}}}\right) d s\right\}\right) \\
& \leq 2 \int_{0}^{t} \beta\left(\left\{\dot{\wp}(t-s) \Theta\left(s ; z_{n} ; u_{z_{n_{x}}}\right)\right\}\right) d s \\
& \leq 2 \int_{0}^{t} \varphi(t-s) \beta\left(\mathcal{P}_{2}\left(D_{0}\right)(s)\right) d s \\
& \leq 2\|\varphi\|_{L^{1}(I)}\left(2(m+1) M \lambda \tau^{\gamma}+M \sum_{i=1}^{m} k_{i}\right) \beta\left(D_{0}\right) .
\end{aligned}
$$


In view of (10), for each $t \in[0, \tau]$, we obtain

$$
\begin{aligned}
\beta\left(\mathcal{P}_{4}\left(D_{0}\right)(t)\right) & =\beta\left(\left\{\int_{0}^{t} \dot{\wp}(t-s)\left(\phi(0)+I\left(s ; z_{n}\right)\right) d s\right\}\right) \\
& \leq 2 \int_{0}^{t} \beta\left(\left\{\dot{\wp}(t-s)\left(\phi(0)+I\left(s ; z_{n}\right)\right)\right\}\right) d s \\
& \leq 2 \int_{0}^{t} \varphi(t-s) \beta\left(\mathcal{P}_{1}\left(D_{0}\right)(s)\right) d s \\
& \leq\left(2\|\varphi\|_{L^{1}(I)} \sum_{i=1}^{m} k_{i}\right) \beta\left(D_{0}\right) .
\end{aligned}
$$

Therefore, by (10), (11), (12) and (13), we can get

$$
\begin{aligned}
\beta\left(\mathcal{P}\left(D_{0}\right)(t)\right) \leq & \beta\left(\mathcal{P}_{1}\left(D_{0}\right)(t)\right)+\beta\left(\mathcal{P}_{2}\left(D_{0}\right)(t)\right)+\beta\left(\mathcal{P}_{3}\left(D_{0}\right)(t)\right)+\beta\left(\mathcal{P}_{4}\left(D_{0}\right)(t)\right) \\
\leq & \sum_{i=1}^{m} k_{i} \cdot \beta\left(D_{0}\right)+\left(2(m+1) M \lambda \tau^{\gamma}+M \sum_{i=1}^{m} k_{i}\right) \beta\left(D_{0}\right) \\
& +2\|\varphi\|_{L^{1}(I)}\left(2(m+1) M \lambda \tau^{\gamma}+M \sum_{i=1}^{m} k_{i}\right) \beta\left(D_{0}\right)+\left(2\|\varphi\|_{L^{1}(I)} \sum_{i=1}^{m} k_{i}\right) \beta\left(D_{0}\right) \\
\leq & \left(1+2\|\varphi\|_{L^{1}(I)}\right)\left(2(m+1) M \lambda \tau^{\gamma}+(1+M) \sum_{i=1}^{m} k_{i}\right) \beta\left(D_{0}\right) \\
\leq & \left(2\left(1+2\|\varphi\|_{L^{1}(I)}\right)(m+1) \lambda M \tau^{\gamma}+\left(1+2\|\varphi\|_{L^{1}(I)}\right)(1+M) \sum_{i=1}^{m} k_{i}\right) \beta\left(D_{0}\right) .
\end{aligned}
$$

Besides, from the equicontinuity of $\mathcal{P}\left(D_{0}\right)$ on each $J_{i}$ and Proposition 7.3 of [31] about the measures of noncompactness, it follows that

$$
\beta\left(\mathcal{P}\left(D_{0}\right)\right)=\max _{0 \leq i \leq m+1} \max _{t \in J_{i}} \beta\left(\left(\mathcal{P} D_{0}\right)(t)\right) .
$$

Consequently, by (7), (14) and (15), we can obtain

$$
\beta\left(D_{0}\right) \leq \beta\left(\overline{c o}\left(\left\{x_{0}\right\} \cup \mathcal{P}\left(D_{0}\right)\right)\right) \leq \beta\left(\mathcal{P}\left(D_{0}\right)\right)<\beta\left(D_{0}\right),
$$

which indicates $\beta\left(D_{0}\right)=0$. By lemma 2 (I)(i), we know that $D_{0}$ is relatively compact. Then from Lemma 3, $\mathcal{P}$ has at least one fixed point $x \in B$, which means that system (1) is exactly controllable on $I$. The conclusion follows.

Remark 3. Resolvent operator is a generalization of $C_{0}$ semigroup and then has more extensive applications [28]. For instance, for the special case that scalar kernel is taken as 1, the resolvent operator $\wp(t)$ becomes the $C_{0}$ semigroup $e^{A t}$ generated by $A$. We refer the readers to [28,32], in which examples are presented to show that they can not generate a $C_{0}$ semigroup but admit a resolvent operator. Then we improve and generalize some analogous results of fractional evolution systems.

\section{Examples}

Example 1. Consider the following fractional partial differential evolution system of the form

$$
\left\{\begin{array}{l}
\frac{\partial^{\frac{3}{5}}}{\partial t^{\frac{3}{5}}} x(t, \xi)=\frac{\partial}{\partial \xi} x(t, \xi)+\frac{\mu(t) x_{t}(\xi)}{1+\left|x_{t}(\xi)\right|}+\int_{-b}^{t} \sigma(t-s) x(s, \xi) d s+\delta(\xi) \omega(t, \xi),(t, \xi) \in[0, a] \times(0,1), \\
x(t, 0)=x(t, 1)=0, t \in[0, a], \\
\Delta x\left(t_{i}, \xi\right)=\frac{\sin \left(x\left(t_{i}, \xi\right)\right)}{2+\zeta_{i}\left(1+\left|x\left(t_{i}, \xi\right)\right|\right)}, i=1,2, \cdots, m, \\
x(t, \xi)=\phi(t, \xi), \quad(t, \xi) \in[-b, 0] \times[0,1],
\end{array}\right.
$$


where $\mu \in C([0, a], R), \sigma \in L([-b, a+b], R), \delta$ is a characteristic function of certain subinterval $D \subset[0,1], \omega \in C([0, a] \times[0,1], R), \zeta_{i} \in C\left(R, R^{+}\right), i=1,2, \cdots, m$, and $\phi \in C([-b, 0] \times$ $[0,1], R)$ which satisfies $\phi(t, 0)=\phi(t, 1)=0$ for $t \in[-b, 0]$.

Define $X=C([0,1], R), \mathcal{D}=\left\{x \in X: x^{\prime} \in X, x(0)=x(1)=0\right\}, A x=x^{\prime}$ for $x \in \mathcal{D}$. Thus, $A$ is an infinitesimal generator of a noncompact semigroup $\{T(t): t \geq$ $0\}$ which is given by $T(t) x(s)=x(t+s)$ for $x \in X$. From the subordinate principle (see Chapter 3, [33]), it follows that $A$ is the infinitesimal generator of a strongly continuous differentiable bounded linear operators family $\{\wp(t)\}_{t \geq 0}(0<\gamma<1)$ such that $\wp(0)=I$, and

$$
\wp(t)=\int_{0}^{\infty} \varphi_{t, \gamma}(s) T(s) d s, t>0,
$$

where $\varphi_{t, \gamma}(s)=t^{-\gamma} \Phi_{\gamma}\left(s t^{-\gamma}\right)$, and

$$
\Phi_{\gamma}(z)=\sum_{n=0}^{\infty} \frac{(-z)^{n}}{n ! \Gamma(-\gamma n+1-\gamma)}=\frac{1}{2 \pi i} \int_{\Gamma_{0}} \varrho^{\gamma-1} \exp \left(\varrho-z \varrho^{\gamma}\right) d \varrho, 0<\gamma<1,
$$

where $\Gamma_{0}$ is a contour which starts and ends at $-\infty$ and encircles the origin once counterclockwise.

$$
\begin{aligned}
& \text { Let } \\
& D^{\frac{3}{5}} x(t)(\xi)=\frac{\partial^{\frac{3}{5}}}{\partial t^{\frac{3}{5}}} x(t, \xi), \\
& x(t)(\xi)=x(t, \xi), \\
& B u(t)(\xi)=\delta(\xi) \omega(t, \xi), \\
& \phi(t)(\xi)=\phi(t, \xi), \\
& f\left(t, x(t), x_{t}\right)(\xi)=\frac{\mu(t) x_{t}(\xi)}{1+\left|x_{t}(\xi)\right|}+\int_{-b}^{t} \sigma(t-s) x(s, \xi) d s, \\
& I_{i}\left(x\left(t_{i}\right)\right)(\xi)=\frac{\sin \left(x\left(t_{i}, \xi\right)\right)}{2+\zeta_{i}\left(1+\left|x\left(t_{i}, \xi\right)\right|\right)}, i=1,2, \cdots, m .
\end{aligned}
$$

Then problem (16) can be regarded as

$$
\left\{\begin{array}{l}
D^{\gamma} x(t)=A x(t)+f\left(t, x(t), x_{t}\right)+B u(t), \text { a.e. } t \in I:=[0, a], \\
\Delta x\left(t_{i}\right)=x\left(t_{i}^{+}\right)-x\left(t_{i}^{-}\right)=I_{i}\left(x\left(t_{i}\right)\right), \quad i=1,2, \cdots, m, \\
x(t)=\phi(t), \quad t \in[-b, 0],
\end{array}\right.
$$

and it is not difficult to check that all the hypotheses of Theorem 1 are satisfied. Then system (16) satisfies exact controllability on $[0, a]$.

Example 2. consider the following fractional partial differential evolution system of the form

$$
\left\{\begin{array}{l}
\frac{\partial^{\frac{1}{3}}}{\partial t^{\frac{1}{3}}} x(t, y)=\Delta x(t, y)+\frac{e^{-2 t}}{3+e^{t}} x(t+\theta, y)+\delta(y) u(t, y), \quad(t, y) \in[0, a] \times \Omega, \\
x(t, y)=0, \quad(t, y) \in[0, a] \times \partial \Omega, \\
\Delta x\left(t_{i}, y\right)=\frac{\sin \left(x\left(t_{i}, y\right)\right)}{e+\omega_{i}\left(1+\left|x\left(t_{i}, y\right)\right|\right)}, i=1,2, \cdots, m, \\
x(t, y)=\phi(t, y), \quad(t, y) \in[-b, 0] \times \Omega,
\end{array}\right.
$$

where $\Omega \subset R^{N}$ represents a bounded domain with smooth boundary $\partial \Omega, \Delta$ denotes the Laplace operator, $\delta$ stands for the characteristic function of certain subdomain $D \subset \Omega, u \in L^{2}([0, a] \times \Omega)$, $\omega_{i} \in C\left(R, R^{+}\right), i=1,2, \cdots, m, \phi \in C^{2,1}([-b, 0] \times \bar{\Omega})$ which satisfies $\phi(t, y) \equiv 0$ for $(t, y) \in$ $[-b, 0] \times \partial \Omega$, and $\theta \in[-b, 0]$.

Let $X=L^{P}(\Omega)$ and the operator $A: \mathcal{D} \subset X \rightarrow X$ defined as $A x=\Delta x$ with domain $\mathcal{D}=\left\{W^{2, N}(\Omega) \cap W_{0}^{1, N}(\Omega)\right\}$. Then, $A$ generates a uniformly bounded analytic semigroup. 
Define $\gamma=\frac{1}{3}, x(t)(y)=x(t, y), u(t)(y)=u(t, y), B u(t)(y)=\delta(y) u(t, y), \phi(t)(y)=\phi(t, y)$, and

$$
I_{i}\left(x\left(t_{i}\right)\right)(y)=\frac{\sin \left(x\left(t_{i}, y\right)\right)}{e+\omega_{i}\left(1+\left|x\left(t_{i}, y\right)\right|\right)}, i=1,2, \cdots, m .
$$

Let

$$
f\left(t, x(t), x_{t}\right)(y)=\frac{e^{-2 t}}{3+e^{t}} x(t+\theta, y) .
$$

Then problem (17) can be regarded as

$$
\left\{\begin{array}{l}
D^{\gamma} x(t)=A x(t)+f\left(t, x(t), x_{t}\right)+B u(t), \text { a.e. } t \in I:=[0, a] \\
\Delta x\left(t_{i}\right)=x\left(t_{i}^{+}\right)-x\left(t_{i}^{-}\right)=I_{i}\left(x\left(t_{i}\right)\right), \quad i=1,2, \cdots, m, \\
x(t)=\phi(t), \quad t \in[-b, 0]
\end{array}\right.
$$

It is not difficult to check that all the hypotheses of Theorem 1 are satisfied. Then system (17) satisfies exact controllability on $[0, a]$.

\section{Conclusions}

This paper derives some new controllability results for a class of fractional impulsive evolution equations with time delay in Banach spaces by using resolvent operator theory and the theory of nonlinear functional analysis. In detail, from the point of view of the restrictions imposed on nonlinearity and impulse terms, exact controllability of the addressed system can be guaranteed even if the nonlinearity and impulse items are only continuous rather than Lipschitz continuous and other restrictive conditions. In order to avoid the limitation that the exact controllability only apply to the finite dimensional space due to the compact semigroup, we substitute the differentiability of resolvent operator for the compactness of semigroup in the present work. With the properly defined delay item in a corresponding complete space, we have solved the disturbances of time delay to the investigation of exact controllability for the considered system.

Further investigation about the nonlocal controllability for a class of fractional impulsive integrodifferential evolution inclusions with time delay and nonlocal conditions will be carried on:

$$
\left\{\begin{array}{l}
D^{\gamma} x(t) \in A x(t)+f\left(t, x_{t}, \int_{0}^{t} k\left(t, s, x_{s}\right) d s\right)+B u(t), \text { a.e. } t \in I:=[0, a] \\
\Delta x\left(t_{i}\right)=x\left(t_{i}^{+}\right)-x\left(t_{i}^{-}\right)=I_{i}\left(x\left(t_{i}\right)\right), i=1,2, \cdots, m \\
x(t)+g_{t}(x)=\phi(t), \quad t \in[-b, 0]
\end{array}\right.
$$

where $g_{t}: C([-b, a], E) \rightarrow E$ is given function. Compared with the classical initial condition $x(0)=x_{0}$ and other nonlocal items $[25,34,35]$, this nonlocal condition has better application effect in physics. In practical applications, it may be given by $g_{t}(x)=\sum_{i=1}^{k} c_{i} x\left(\tau_{i}+t\right), t \in$ $[-b, 0]$, where $c_{i}(i=1,2, \cdots, k)$ are given constants and $0<\tau_{1}<\tau_{2}<\cdots<\tau_{n} \leq a$. At time $t=0$, we have $g_{0}(x)=\sum_{i=1}^{k} c_{i} x\left(\tau_{i}\right)$, which is exactly the cases in $[25,34,35]$.

Funding: This research was funded by the National Natural Science Foundation of China under grant 61873150, the Young Experts of Taishan Scholar Project under grant tsqn201909076, and a project of Shandong Province Higher Educational Science and Technology Program of China under the grant J18KA233.

Institutional Review Board Statement: Not applicable.

Informed Consent Statement: Not applicable.

Data Availability Statement: Not applicable.

Conflicts of Interest: The author declares no conflict of interest. 


\section{References}

1. Zhao, D.; Liu, Y. Positive solutions for a class of fractional differential coupled system with integral boundary value conditions. J. Nonlinear Sci. Appl. 2016, 9, 2922-2942. [CrossRef]

2. Li, L.; Jiang, Z.; Yin, Z. Compact finite-difference method for 2D time-fractional convection-diffusion equation of groundwater pollution problems. Comput. Appl. Math. 2020, 39, 1-34. [CrossRef]

3. Mao, J.; Zhao, D. Multiple positive solutions for nonlinear fractional differential equations with integral boundary value conditions and a parameter. J. Funct. Space 2019, 2019, 2787569. [CrossRef]

4. Saleem, N.; Iqbal, I.; Iqbal, B.; Radenovíc, S. Coincidence and fixed points of multivalued F-contractions in generalized metric space with application. J. Fixed Point Theory Appl. 2020, 22, 81. [CrossRef]

5. Zhao, D.; Liu, Y. Twin solutions to semipositone boundary value problems for fractional differential equations with coupled integral boundary conditions. J. Nonlinear Sci. Appl. 2017, 10, 3544-3565. [CrossRef]

6. Liu, Y. Bifurcation techniques for a class of boundary value problems of fractional impulsive differential equations. J. Nonlinear Sci. Appl. 2015, 8, 340-353. [CrossRef]

7. Vijayakumar, V.; Selvakumar, A.; Murugesu, R. Controllability for a class of fractional neutral integro-differential equations with unbounded delay. Appl. Math. Comput. 2014, 232, 303-312. [CrossRef]

8. Zhao, D.; Liu, Y. Eigenvalues of a class of singular boundary value problems of impulsive differential equations in Banach spaces. J. Funct. Space 2014, 2014, 720494. [CrossRef]

9. Ji, S.; Li, G.; Wang, M. Controllability of impulsive differential systems with nonlocal conditions. Appl. Math. Comput. 2011, 217, 6981-6989. [CrossRef]

10. Wang, J.; Zhou, Y. Complete controllability of fractional evolution systems. Commun. Nonlinear Sci. Numer. Simulat. 2012, 17, 4346-4355. [CrossRef]

11. Du, J.; Jiang, W.; Pang, D.; Niazi, A.U.K. Controllability for a new class of fractional neutral integro-differential evolution equations with infinite delay and nonlocal conditions. Adv. Differ. Equ. 2017, 139, 1-22. [CrossRef]

12. Tai, Z. Controllability of fractional impulsive neutral integrodifferential systems with a nonlocal Cauchy condition in Banach spaces. Appl. Math. Lett. 2011, 24, 2158-2161. [CrossRef]

13. Zhao, D.; Liu, Y.; Li, X. Controllability for a class of semilinear fractional evolution systems via resolvent operators. Commun. Pur. Appl. Anal. 2019, 18, 455-478. [CrossRef]

14. Balachandran, K.; Park, J.Y. Controllability of fractional integrodifferential systems in Banach spaces. Nonlinear Anal. 2009, 3, 363-367. [CrossRef]

15. Debbouche, A.; Baleanu, D. Controllability of fractional evolution nonlocal impulsive quasilinear delay integro-differential systems. Comput. Math. Appl. 2011, 62, 1442-1450. [CrossRef]

16. Zhao, D. New results on controllability for a class of fractional integrodifferential dynamical systems with delay in Banach spaces. Fractal Fract. 2021, 5, 1-18. [CrossRef]

17. Nirmala, R.J.; Balachandran, K.; Rodríguez-Germa, L.; Trujillo, J.J. Controllability of nonlinear fractional delay dynamical systems. Rep. Math. Phys. 2016, 77, 87-104. [CrossRef]

18. Zhao, D.; Liu, Y. Controllability of nonlinear fractional evolution systems in Banach spaces: A survey. Electron. Res. Arch. 2021, 29, 3551-3580. [CrossRef]

19. Li, K.; Peng, J.; Gao, J. Controllability of nonlocal fractional differential systems of order $\alpha \in(1,2]$ in Banach spaces. Rep. Math. Phys. 2013, 71, 33-43.

20. Zhao, D.; Liu, Y.; Li, H. Fast-time complete controllability of nonlinear fractional delay integrodifferential evolution equations with nonlocal conditions and a parameter. Math. Meth. Appl. Sci. 2021. [CrossRef]

21. Tai, Z.; Lun, S. On controllability of fractional impulsive neutral infinite delay evolution integrodifferential systems in Banach spaces. Appl. Math. Lett. 2012, 25, 104-110. [CrossRef]

22. Tai, Z.; Wang, X. Controllability of fractional-order impulsive neutral functional infinite delay integrodifferential systems in Banach spaces. Appl. Math. Lett. 2009, 22, 1760-1765. [CrossRef]

23. Yan, Z. Controllability of fractional-order partial neutral functional integrodifferential inclusions with infinite delay. J. Franklin Inst. 2011, 348, 2156-2173. [CrossRef]

24. El-Borai, M.M. Some probability densities and fundamental solutions of fractional evolution equations. Chaos Solitons Fract. 2002, 14, 433-440. [CrossRef]

25. Liang, J.; Yang, H. Controllability of fractional integro-differential evolition equations with nonlocal conditions. Appl. Math. Comput. 2015, 254, 20-29.

26. Ravichandran, C.; Baleanu, D. On the controllability of fractional functional integro-differential systems with an infinite delay in Banach spaces. Adv. Differ. Equ. 2013, 291,1-13. [CrossRef]

27. Podlubny, I. Fractional Differential Equations, Mathematics in Science and Engineering; Academic Press: New York, NY, USA, 1999.

28. Prüss, J. Evolutionary Integral Equations and Applications. In Monographs in Mathematics; Birkhäuser: Basel, Switzerland, 1993; Volume 87.

29. Guo, D.; Lakshmikantham, V.; Liu, X. Nonlinear Integral Equations in Abstract Spaces; Kluwer Academic Publishers: New York, NY, USA, 1996. 
30. Agarwal, R.P.; Benchohra, M.; Hamani, S. A survey on the existence results for boundary value problems of nonlinear fractional differential equations and inclusions. Acta Appl. Math. 2010, 109, 973-1033. [CrossRef]

31. Deimling, K. Nonlinear Functional Analysis; Springer: Berlin, Germany, 1985.

32. Grimmer, R.; Prüss, J. On linear Volterra equations in Banach spaces. J. Comp. Appl. Math. 1985, 11, 189-205. [CrossRef]

33. Bazhlekova, E. Fractional Evolution Equations in Banach Spaces; University Press Facilities, Eindhoven University of Technology: Eindhoven, The Netherlands, 2001.

34. Deng, K. Exponential decay of solutions of semilinear parabolic equations with nolocal initial conditions. J. Math. Anal. Appl. 1993, 179, 630-637. [CrossRef]

35. Byszewski, L. Existence and uniqueness of classical solutions to a functional defferential abstact nonlocal Cauchy problem. J. Math. Appl. Stoch. Anal. 1999, 12, 91-97. [CrossRef] 